SABIC

3D-GEFORMTE LEXAN-PLATTEN FÜR DIE FAHRZEUGVERGLASUNG

Bediener von Forstwirtschaftsfahrzeugen sind bei ihrer Arbeit im Wald auf eine uneingeschränkte Sicht und den Schutz gegen Bruchstücke oder lange Objekte wie Äste oder Maschinenteile angewiesen, die in ihre Kabinen eindringen können. Die Rottne Industry $A B$ wählte für die Verscheibung seiner Holzvollernter den Kunststoff Lexan Margard Polycarbonat mit hoher optischer Qualität von Sabic. Seit Verwendung der Lexan-Platten sei die optische Qualität der Windschutzscheiben und Fenster gestiegen, zitiert Sabic seinen Kunden. Die Bediener bestätigten die klarere und schärfere Sicht aus der Kabine.

Lexan Margard MR5E-Platten sind beidseitig hartbeschichtete Platten mit UV-Schutz. Die Platte sei optional mit einer harten, im Siebdruckverfahren angebrachten Schutzbeschichtung für 3D-geformte Komponenten erhältlich und verbessere die Sicherheit dieser Anwen- dungen mit industrieführenden optischen Eigenschaften, wie einer hohen Schlagzähigkeit. Die Platten absorbieren nach Angaben von Sabic infrarotnahe (IR) Lichtstrahlung und steigern zusammen mit hohem UV-Schutz sowie hoher Beständigkeit gegen Verschleiß und Schläge den Komfort und den Schutz der Bediener bei heißem Wetter. „Beide branchenführenden Produkte werden in der neuen Fertigungsstraße mit Extrusionsverfahren für Platten im Sabic-Werk in Bergen op Zoom in den Niederlanden hergestellt", heißt es.

Die Lexan-Platten mit Stärken zwischen 2 und $15 \mathrm{~mm}$ eigneten sich für die Laminierung und Verscheibung von Forst- wirtschafts- und Landwirtschaftsmaschinen, Hochgeschwindigkeitszügen, kugelsicheren Glaslaminaten aus PC für VIP-, Militär und Polizeifahrzeugen sowie für die Verscheibung in Hochsicherheitseinrichtungen.

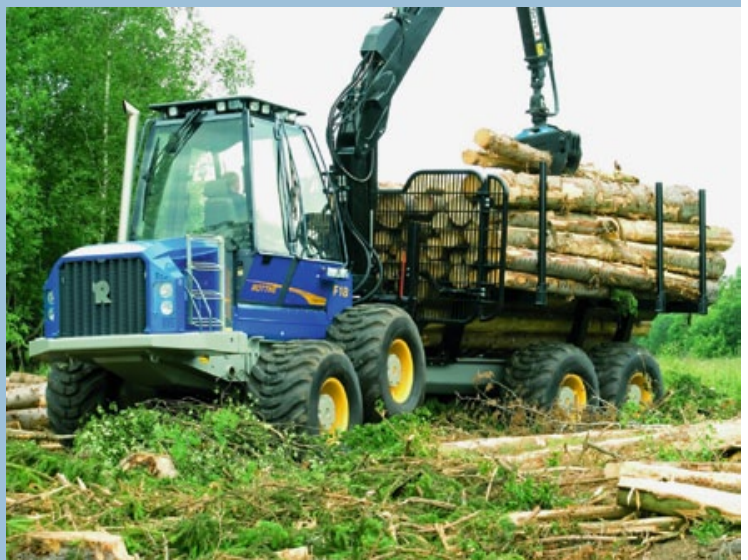

VICTREX

\title{
PEEK-Folien für die Luft- und Raumfahrt
}

Gewichtsreduktion und Effizienzsteigerungen führen dazu, dass Hersteller der Luftund Raumfahrtindustrie bei Strukturbauteilen Aluminium mehr und mehr durch Composite-Bauteile ersetzen. „Beim Kontakt von Karbonfasern mit Kupfer oder Alu-

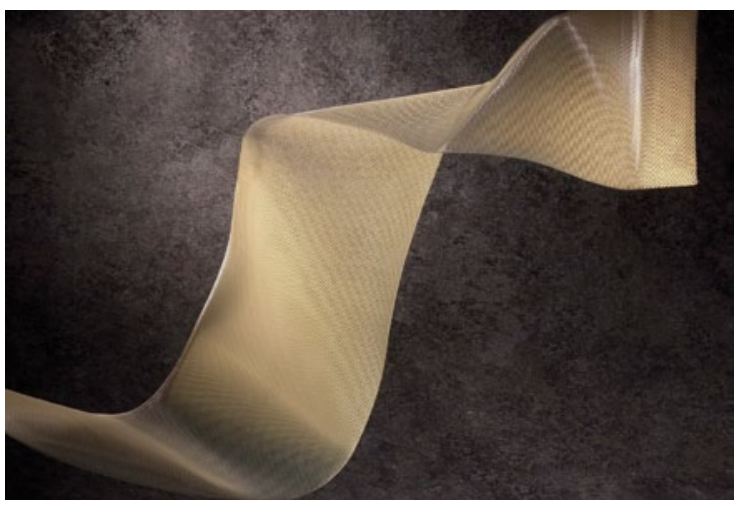

minium tritt jedoch eine galvanische Reaktion ein, das Material korrodiert. Um die Korrosion zu reduzieren, suchen Konstruktionsingenieure nach unterschiedlichen Polymeren als Barriereschicht zwischen der Karbonfaser und dem expandierten Material“, erklärt Ken Burtt, Vice President Sales and Marketing beim US-amerikanischen Hersteller von expandierten Metall- und Kunststofffolien Dexmet. APTIV-Folien auf Grundlage des Hochleistungskunststoffs Victrex PEEK seien eine Option, weil sie dünn und hochfest sind. Sie verfügten zusätzlich über exzellente Hochtemperatur- und dielektrische Eigenschaften. Bei der Fertigung der Kohlenstofffaser-Bauteile seien auch die Hochtemperatureigenschaften von zentraler Bedeutung. „Hier auf expandierte APTIVFolien zu setzen, ist sogar die bessere Option aufgrund ihres niedrigeren Gewichts", heißt es weiter.

Seit mehr als 60 Jahren stellt die Dexmet Corporation Präzisionsstreckmetalle her und hat Lösungen beim Expandieren von dünnen Folien aus Metallen oder Polymeren erzielt, die sich durch exakte mechanische und elektrische Eigenschaften auszeichnen und so die Anforderungen an Leitfähigkeit, Gewicht und Maßtoleranzen erfüllen.

Victrex plc. mit Hauptsitz in Großbritannien ist nach eigenen Angaben weltweit führender Hersteller von Hochleistungspolymeren, zu denen auch Victrex PEEK Polymer, APTIVFolien, VICOTE-Beschichtungen und VictrexPipes zählen. 